(A. N. 4404) oder Nr. 45 (A. N. 4478) wäre gerade oder beinahe Raum genug. Von sämtlichen (6) Maxima gelang es, sowohl den Aufstieg als den Abstieg zu beobachten, so daß der Typus der Maxima sicher bestimmt werden konnte.

Die diesjährige Lichtkurve zeigt eine regelmäßige $\mathrm{Ab}$ wechslung von langen und kurzen Maxima mit zwischenliegenden Perioden von nahezu konstanter Helligkeit. Nur einmal wurde $z$ wischen den Maxima eine schwache Aufhellung beobachtet: am 9.Juni (J.D. 24I9I97) hatte sich
SS Cygni langsam von $\mathrm{I} \mathrm{I}^{\mathrm{m}} \cdot 4$ auf I I ${ }^{\mathrm{m}} \mathrm{I}$ gehoben, sank dann aber in zwei Tagen wieder auf $\mathrm{II}^{\mathrm{m}} \cdot 4$ herab.

Hier folgt eine Übersicht des Jahres I9I I. Außer dem Maximum ist wieder der Moment gegeben, in dem SS Cygni beim Aufstieg die Helligkeit $9^{m} \cdot 35$ erreichte. Unter "Dauer " ist, wie früher, zu verstehen, wieviel Tage der Veränderliche heller als $I \mathbf{I}^{\mathrm{m}} \circ$ $\circ$ war; auch die unter $\mathrm{Bb}$. angeführte Zahl der Beobachtungen bezieht sich auf die Helligkeit $>\mathbf{I} \mathbf{I}^{\mathrm{m}}$.o. Die Zeit ist $\mathrm{m}$. Z. Utrecht.

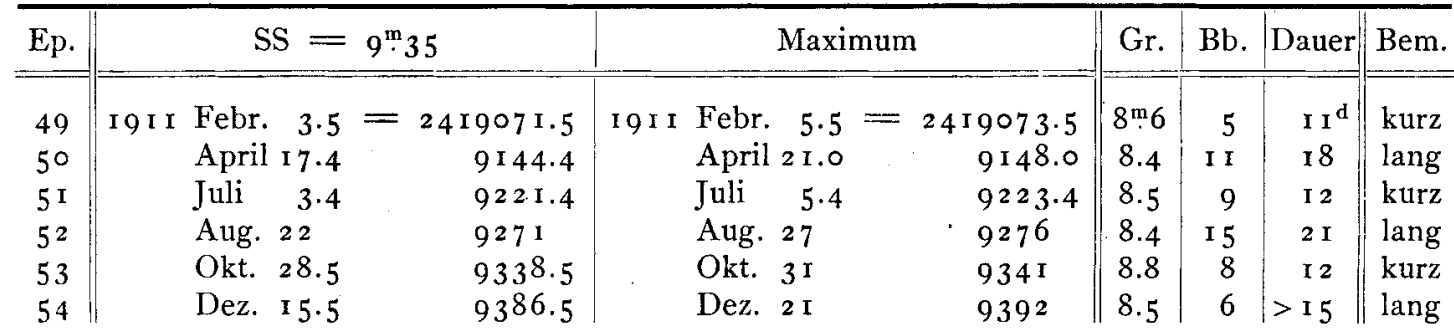

Merkwürdig ist die Folge von zwei sehr langen Intervallen des konstanten Lichtes in den ersten Monaten des Jahres I 9 I x. Zwischen Nr. 49 und 50 verliefen 73, zwischen

Nr. 50 und $5 \mathrm{I}$ nicht weniger als 77 Tage; möglich bleibt allerdings, wie oben erwähnt, ein sehr kurzes Maximum zwischen dem 9. und dem 17. März.

Utrecht, I 9 I 2 Januar 5.

A. A. Nijland.

\title{
Beobachtungen des Kometen 1911 c (Brooks)
}

am vierzölligen Meridiankreise der Sternwarte $z u$ Königsberg.

\begin{tabular}{|c|c|c|c|c|c|}
\hline I 9 I I & M.Z.Kö & igsberg & & $\alpha$ a & \\
\hline Aug. 20 & $\mathrm{II}^{\mathrm{h}} \mathrm{I} 3$ & 19.4 & $2 \mathrm{I}^{\mathrm{h}}$ & & 52.22 \\
\hline 2 I & I I & 0.7 & 20 & 59 & 49.82 \\
\hline 24 & 1035 & 18.3 & 20 & 42 & 50.97 \\
\hline 27 & 10 & 40.3 & 20 & 22 & 35.57 \\
\hline 28 & $5 \mathrm{I}$ & 54.2 & 20 & I 5 & 3.45 \\
\hline 30 & $9 \quad 27$ & 42.6 & & - & \\
\hline $3 \mathrm{I}$ & I 4 & 54.8 & I 9 & 49 & 45.60 \\
\hline Sept. & I 9 & 8. I & I 9 & & 33.48 \\
\hline
\end{tabular}

\begin{tabular}{|c|c|c|}
\hline & app. & \\
\hline $42^{\circ}$ & $59^{\prime}$ & 57 \\
\hline 43 & 5.3 & 42 \\
\hline 46 & 34 & 6 \\
\hline-49 & I I & 4 \\
\hline 50 & $\mathbf{I}$ & $5 \mathrm{I}$ \\
\hline $5 \mathrm{I}$ & $3^{8}$ & 47 \\
\hline $5^{2}$ & 24 & 54 \\
\hline 55 & & \\
\hline
\end{tabular}

$\log p \cdot \Delta \quad$ Red. ad l. app.

$0.247+2^{5} .42+8.20$

$0.2 \mathrm{I} 2+2.4 \mathrm{I}+8.49$

$0.088+2.36+9.36$

$9.916+2.28+10.25$

$9.84 \mathrm{I}+2.24+10.53$

$9.649-\quad+$ II.06

$9.515+2.07+11.31$

$8.906 \mathrm{n}+\mathrm{I} .75+\mathrm{I}$ I.99

Aug. 20. Äußerst schwach, sehr unsicher. - Aug. 2 I. Dunstig, Komet sehr schwach, ohne Kern. - Aug. 28. Sehr schwach, dunstig. - Aug. 30. Für Rektaszensionsbeobachtung zu schwach, nur geahnt. - Aug. 3 I. Unsicher.

Der Komet ist lediglich während des Durchgangs durch die hier beobachtete Deklinationszone mitgenommen worden, im ganzen bot seine damals ziemlich kernlose Erscheinung kein geeignetes Objekt für eine Beobachtung am Meridiankreis.

Königsberg, I 9 I 2 Januar.

E. Fost.

\section{Helligkeitsschätzungen des Kometen 1911 c (Brooks). Von L. Filippoff.}

In Ergänzung zu der kurzen Bemerkung (A. N. 4542) über meine Helligkeitsschätzungen des Kometen 19 I I $c$ teile ich jetzt ausfuhrlich alle von mir gemachten Beobachtungen mit. Sie erstrecken sich auf die Zeit von I9 I I Aug. I 7 bis Okt. 3 .

Die Beobachtungen wurden in der Regel, wie ich schon angegeben habe, mit einem Zeißschen Prismenbinokel mit 8 facher Vergrößerung gemacht, bei derartig unscharfer Okularstellung (hinter dem Brennpunkt), daß der Kern des Kometen und die Vergleichsterne als fast gleich große Scheiben erschienen, wodurch eine größere Genauigkeit der Vergleichungen der relativen Helligkeit des Kometen und der benachbarten Vergleichsterne erreicht wurde.

$\mathrm{Nach}$ dem 20. September wurden die Vergleichungen teilweise mit bloßem Auge gemacht. In der Zusammenstellung der Beobachtungen sind diese Vergleichungen durch ein $\dagger$ bei dem Werte der beobachteten Helligkeit des Kometen bezeichnet.
Die folgende Tabelle enthält die Vergleichsterne und ihre der PD entnommenen Helligkeiten.

$$
\begin{aligned}
& \text { Name } \\
& \mathrm{BD}+40^{\circ} 4485 \\
& \text { " }+39^{\circ} 4529 \\
& "+42^{\circ} 4046 \\
& \text { 》+61 }{ }^{\circ} 2109 \\
& >+50^{\circ} 3067 \\
& "+47^{\circ} 3 \text { r } 88 \\
& \text { " }+44^{\circ} 3670 \\
& \begin{array}{r}
4403679 \\
+55^{\circ} 193
\end{array} \\
& +58^{\circ} 2201 \\
& \psi \text { Cygni } \\
& d \gg \\
& x \text { » }
\end{aligned}
$$

\begin{tabular}{|c|c|c|c|}
\hline$H$ & * & & Name \\
\hline $6 \mathrm{~m} \cdot 28$ & I 3 & & raconis \\
\hline 6.73 & I 4 & $d$ & $»$ \\
\hline 6.36 & I 5 & $c$ & » \\
\hline 7.60 & I 6 & $\nu$ & $»$ \\
\hline 7.24 & I 7 & $\xi$ & $\gg$ \\
\hline 5.70 & I 8 & $y$ & $》$ \\
\hline 6.34 & I 9 & $\eta$ & $»$ \\
\hline 5.19 & 20 & $i$ & $»$ \\
\hline $5 \cdot 59$ & $2 \mathrm{I}$ & & ootis \\
\hline $5.1 \mathrm{I}$ & 22 & $\gamma$ & $\gg$ \\
\hline 5.12 & 23 & $\alpha$ & oronae \\
\hline 4.02 & 24 & $\gamma$ & rsae mi \\
\hline
\end{tabular}

\section{Vergleichsterne.}

$H$
$4 \cdot 80$
5.00
5.29
5.18
3.96
4.26
3.07
$4 \cdot 79$
$3 \cdot 72$
$3 \cdot 36$
2.60
3.36

